PROCEEDINGS OF THE

AMERICAN MATHEMATICAL SOCIETY

Volume 132, Number 8, Pages 2327-2329

S 0002-9939(04)07354-X

Article electronically published on February 13, 2004

\title{
FUNCTIONAL ANALYSIS PROOFS OF ABEL'S THEOREMS
}

\author{
M. T. KARAEV
}

(Communicated by Joseph A. Ball)

\begin{abstract}
We give alternative proofs to the classical theorems of Abel, using the concept of Berezin symbol.
\end{abstract}

1.

Let $\left\{a_{n}\right\}_{n=0}^{\infty}$ be a sequence of complex numbers. The sequence $\left\{a_{n}\right\}_{n=0}^{\infty}$ is Abel convergent (written $(A)$ convergent) to $a$ if the limit

$$
\lim _{t \rightarrow 1^{-}}(1-t) \sum_{n=0}^{\infty} a_{n} t^{n}=a
$$

exists. The series $\sum_{n=0}^{\infty} a_{n}$ is $(A)$ convergent to $L$ if the sequence of partial sums $\left\{s_{n}\right\}_{n=0}^{\infty}$ (where $\left.s_{n} \stackrel{\text { def }}{=} \sum_{k=0}^{n} a_{k}\right)$ is $(A)$ convergent to $L$.

The following famous results are due to Abel (e.g., see 1], [2]).

Theorem 1 (Theorem of Abel). If $\left\{a_{n}\right\}_{n=0}^{\infty}$ converges to $a$, then $\left\{a_{n}\right\}_{n=0}^{\infty}(A)$ converges to $a$.

Theorem 2 (Theorem of Abel). If the series $\sum_{n=0}^{\infty} a_{n}$ converges to $L$, then $\sum_{n=0}^{\infty} a_{n}$ is $(A)$ convergent to $L$.

This paper presents functional analysis proofs of these results. To give our proofs, we first define what is meant by a Berezin symbol.

2 .

Let $H^{2}$ denote the Hardy space of functions analytic on the unit disk $\mathbb{D}=$ $\{z \in \mathbb{C}:|z|<1\}$. For a general bounded operator $A$ on the Hardy space, the Berezin symbol of $A$ is (see [3] ) the function $\tilde{A}$ defined by

$$
\tilde{A}(\lambda)=\left(A \hat{k}_{\lambda}, \hat{k}_{\lambda}\right), \lambda \in \mathbb{D}
$$

Received by the editors March 3, 2003 and, in revised form, April 28, 2003.

2000 Mathematics Subject Classification. Primary 47A15.

Key words and phrases. Abel convergent, Berezin symbol, diagonal operator.

(C)2004 American Mathematical Society 
where $\hat{k}_{\lambda}$ is the normalized reproducing kernel of $H^{2}$. The function $\hat{k}_{\lambda}$ is defined by

$$
\hat{k}_{\lambda}(z)=\frac{\sqrt{1-|\lambda|^{2}}}{1-\bar{\lambda} z}
$$

for $z \in \mathbb{D}$ and has the property that $\left(f, \hat{k}_{\lambda}\right)=\sqrt{1-|\lambda|^{2}} f(\lambda)$, for $f \in H^{2}$, and this obviously approaches 0 for $f \in H^{\infty}$ (the space of all bounded analytic functions on $\mathbb{D}$ ), and hence for all $f \in H^{2}$, whenever $|\lambda| \rightarrow 1^{-}$. Thus, the kernels $\hat{k}_{\lambda}$ converge weakly to zero as $\lambda$ approaches the unit circle $\partial \mathbb{D}$ (i.e., the Hardy space $H^{2}$ is standard [3]). Then we have that if $A$ is a compact operator on $H^{2}$, then $\tilde{A}(\lambda) \rightarrow 0$ as $\lambda \rightarrow \partial \mathbb{D}$. In this sense, the Berezin symbol of a compact operator on $H^{2}$ vanishes on the boundary.

3.

Proof of Theorem 1 . Let us consider the diagonal operator $\mathcal{D}_{\left\{a_{n}\right\}}$ on $H^{2}$ defined by

$$
\mathcal{D}_{\left\{a_{n}\right\}} z^{k}=a_{k} z^{k}, \quad k=0,1,2, \ldots .
$$

Since $\left\{a_{n}\right\}$ is a bounded sequence, $\mathcal{D}_{\left\{a_{n}\right\}}$ is a bounded operator on $H^{2}$. We now calculate the Berezin symbol of an operator $\mathcal{D}_{\left\{a_{n}\right\}}$. We have

$$
\begin{aligned}
\tilde{\mathcal{D}}_{\left\{a_{n}\right\}}(\lambda) & =\left(\mathcal{D}_{\left\{a_{n}\right\}}, \hat{k}_{\lambda}, \hat{k}_{\lambda}\right)=\sqrt{1-|\lambda|^{2}}\left(\mathcal{D}_{\left\{a_{n}\right\}} \sum_{k=0}^{\infty} \bar{\lambda}^{k} z^{k}, \hat{k}_{\lambda}\right) \\
& =\sqrt{1-|\lambda|^{2}}\left(\sum_{k=0}^{\infty} \bar{\lambda}^{k} \mathcal{D}_{\left\{a_{n}\right\}} z^{k}, \hat{k}_{\lambda}\right) \\
& =\sqrt{1-|\lambda|^{2}}\left(\sum_{k=0}^{\infty} \bar{\lambda}^{k} a_{k} z^{k}, \hat{k}_{\lambda}\right) \\
& =\left(1-|\lambda|^{2}\right) \sum_{k=0}^{\infty} a_{k}|\lambda|^{2 k} .
\end{aligned}
$$

Thus,

$$
\tilde{\mathcal{D}}_{\left\{a_{n}\right\}}(\lambda)=\left(1-|\lambda|^{2}\right) \sum_{k=0}^{\infty} a_{k}|\lambda|^{2 k}, \lambda \in \mathbb{D}
$$

(i.e., $\tilde{\mathcal{D}}_{\left\{a_{n}\right\}}$ is a radial function, $\tilde{\mathcal{D}}_{\left\{a_{n}\right\}}(\lambda)=\tilde{\mathcal{D}}_{\left\{a_{n}\right\}}(|\lambda|)$ ), which yields

$$
\tilde{\mathcal{D}}_{\left\{a_{n}\right\}}(\sqrt{t})=(1-t) \sum_{k=0}^{\infty} a_{k} t^{k}, \quad 0<t<1 .
$$

Then from (10) we have

$$
(1-t) \sum_{k=0}^{\infty} a_{k} t^{k}=(1-t) \sum_{k=0}^{\infty}\left(a_{k}-a\right) t^{k}+a(1-t) \sum_{k=0}^{\infty} t^{k}=\tilde{\mathcal{D}}_{\left\{a_{n}-a\right\}}(\sqrt{t})+a .
$$

Since by the condition of the theorem $a_{n}-a \rightarrow 0$ as $n \rightarrow \infty, \tilde{\mathcal{D}}_{\left\{a_{n}-a\right\}}$ is a compact operator on $H^{2}$. Hence, its Berezin symbol vanishes on the boundary, i.e.,

$$
\lim _{t \rightarrow 1^{-}} \tilde{\mathcal{D}}_{\left\{a_{n}-a\right\}}(\sqrt{t})=0 .
$$


Then the last equality yields

$$
\lim _{t \rightarrow 1^{-}}(1-t) \sum_{k=0}^{\infty} a_{k} t^{k}=a,
$$

which completes the proof.

4.

Proof of Theorem 2. The argument that was used to prove Theorem 1 can easily be modified to prove the equality

$$
\tilde{\mathcal{D}}_{\left\{s_{n}\right\}}(\sqrt{t})=\sum_{k=0}^{\infty} a_{k} t^{k}, \quad 0<t<1 .
$$

Formula (2) implies that for each $t \in(0,1)$ the series $\sum_{k=0}^{\infty} a_{k} t^{k}$ is convergent. On the other hand,

$$
\mathcal{D}_{\left\{s_{n}\right\}}=L I+\mathcal{D}_{\left\{s_{n}-L\right\}},
$$

where the diagonal operator $\mathcal{D}_{\left\{s_{n}-L\right\}}$ is compact since by the hypothesis of the theorem $s_{n}-L \rightarrow 0$ as $n \rightarrow \infty$, and therefore from the formula (2) we have

$$
\begin{aligned}
\lim _{t \rightarrow 1^{-}} \sum_{k=0}^{\infty} a_{k} t^{k} & =\lim _{t \rightarrow 1^{-}} \tilde{\mathcal{D}}_{\left\{s_{n}\right\}}(\sqrt{t}) \\
& =\lim _{t \rightarrow 1^{-}}\left(L+\tilde{\mathcal{D}}_{\left\{s_{n}-L\right\}}(\sqrt{t})\right) \\
& =L+\lim _{t \rightarrow 1^{-}} \tilde{\mathcal{D}}_{\left\{s_{n}-L\right\}}(\sqrt{t}) \\
& =L,
\end{aligned}
$$

which means that the series $\sum_{k=0}^{\infty} a_{k}(A)$ converges to $L$. The theorem is proved.

\section{REFERENCES}

[1] A. Zygmund, Trigonometric series, vol. 1, Cambridge University Press, Cambridge, 1959. MR 21:6498

[2] R. E. Powell and S. M. Shah, Summability theory and applications, Van Nostrand Reinhold, London, 1972.

[3] E. Nordgren and P. Rosenthal, Boundary values of Berezin symbols, Nonselfadjoint Operators and Related Topics (Beer Sheva, 1992), Operator Theory: Advances and Applications, vol. 73, Birkhäuser, Basel, 1994, pp. 362-368. MR 96b:46036

Institute for Mathematics and Mechanics, Azerbaijanian National Academy of Sciences, F.Agaev, 9, 370141 Baku, Azerbaijan

E-mail address: garayev@fef.sdu.edu.tr

Current address: Suleyman Demirel University, Faculty of Arts and Sciences, Department of Mathematics, 32260 Isparta, Turkey 